\title{
Generalized Theory on Direct Arm Energy Control in Modular Multilevel Converters
}

\author{
Milan Utvić and Dražen Dujić
}

\begin{abstract}
Modular multilevel converters have become standard solution in many power demanding high voltage and medium voltage application. Scalability and modularity come with the price of increased control complexity, compared to the other multilevel converter topologies, in a sense that the submodules within the converter have to maintain their voltage level around some predefined value. Intra-arm voltage balancing methods aim to equally distribute charge among the submodules within a single arm, whereas total energy content within each arm has to correspond to the reference value. While most of the literature explores control methods aiming to balance energies among the converter arms, this paper sets the theoretical foundation for the independent control of arm-energies, and presents generalized control concepts that can be applied for the purpose of arm energy control. Proposed control schemes aim to minimize the current stress imposed on the converter, offer easy implementation and be general in a sense of their application to different types of modular multilevel converters. Moreover, they are valid for both balanced and unbalanced grid conditions, as well as in case of failure of one or several submodules within an arm. Theoretical considerations are verified through an extensive set of simulations.
\end{abstract}

Index Terms-Energy balancing, energy control, fault-tolerant operation, modular multilevel converter (MMC), unbalanced grid conditions.

\section{INTRODUCTION}

E VER since its introduction in [1] modular multilevel converter (MMC) has become standard solution in many power demanding applications. The most prominent application of MMC is in high voltage DC (HVDC) transmission systems, where contemporary converter topologies are mostly based on MMC, owing to the advantages of the voltage source converters (VSCs) over the standard line-commutated converters (LCCs), such as independent reactive and active power control, fast transient response, ability to supply weak networks, and black start capability [2]. Apart from the advantages offered by the nature of VSCs, MMC has some additional prominent features, such as the ease of scalability, modularity, redundancy, high efficiency, elimination of the bulky transformers, filters and DC link capacitor [3], making the MMC preferable VSC solution

Manuscript received January 20, 2020; revised June 12, 2020; accepted June 12, 2020. Date of publication December 30, 2020; date of current version December 9,2020

Both authors are with Power Electronics Laboratory, École Polytechnique Fédérale de Lausanne, Vaud, Switzerland (e-mail: milan.utvic@epfl.ch; drazen. dujic@epfl.ch).

Digital Object Identifier 10.24295/CPSSTPEA.2020.00032 for the high voltage, high power transfer. Both academia and industry are showing interest in the application of MMC in various other areas, such as flexible $\mathrm{AC}$ transmission systems (FACTS) [4], [5], breaker-less medium voltage DC (MVDC) shipboard systems [6], MVDC collection grids for offshore wind farms [7], integration of the energy storage systems, etc.

Scalability and modularity of MMCs come with the price of increased control complexity, compared to the other multilevel converter topologies, such as cascaded H-bridge (CHB), in a sense that the submodules (SMs) within an MMC have to maintain their voltage (energy) level around some defined value. Control method that does not need additional layers of energy control is referred to as direct modulation in the literature [8], and presents the simplest control scheme. However, this control approach suffers from the parasitic 2nd harmonic circulating current [8], has rather poor internal dynamic performance [9], contributes to the oscillation in the DC link during the unbalanced grid conditions [10] and can cause parasitic first harmonic arm-common current in case when capacitances of different SMs differ significantly [11]. On the other hand, closed-loop voltage control uses the measured capacitor voltages in order to modify the modulation index, and as a result, this method is not inherently stable [8], [9]. This imposes the need for additional control layers, preventing the divergence of the arm energies from the desired values.

Generally speaking, proper operation of an MMC requires arm energy/voltage control, and intra-arm voltage balancing actions, which aim to equally distribute the charge among SMs within an arm. While methods for the intra-arm voltage balancing are various and extensively studied in the literature [1], [12]-[14], they are decoupled from the arm energy control/ balancing methods, and further not discussed within this paper.

Control schemes ensuring energy balancing among the arms of an MMC were introduced in [8], and have also been well studied in the literature [15]-[21]. Most of the proposed schemes focus on equal distribution of the energy content among the arms of an MMC, which is not always the control objective. Namely, due to its redundancy, modularity and good dynamic performance, $\mathrm{MMC}$ is perceived as a robust converter, able to remain in operation under various undesirable effects, such as tolerances in the components, failure of one or several SMs within an arm, unbalanced grid conditions, etc. Failure of one or several SMs within an arm should not alter normal operation of an MMC, however the energy content of the corresponding arm changes accordingly. Therefore, contrary to the arm energy balancing methods, this paper introduces the direct arm energy 
control (DAEC) method, which enables independent control of the arm energies. In addition, owing to the per-phase based approach of the arm energy control, proposed control schemes are easily applicable to an MMC operating under unbalanced grid conditions, $\mathrm{MMC}$ with arbitrary number of phase-legs, MMC with an arbitrary number of paralleled arms, as well as to the modular multilevel matrix converter (MMMC). Finally, implementation of the presented control schemes is easy and intuitive, and ensures minimal current stress imposed on the converter during the energy control actions.

In this paper, theoretical foundation for the direct arm energy control within an MMC is set, energy control mechanisms are identified, and appropriate control schemes are presented. As a showcase, three-phase (3PH) MMC is used, where direct control of arm energies is achieved through the so-called armdifferential $(\Delta)$ and arm-sum $(\Sigma)$ energy control mechanisms. Possibility to apply presented control schemes to other variants of an MMC are also discussed within the paper.

This paper is organized as follows: Section II identifies the need for the direct control of arm-energies of an MMCs, defines control variables for their control, introduces two control mechanisms and briefly covers basic operational principles of an MMC. Section III sets the theoretical foundation for the arm-differential energy control, proposes two possible approaches, and presents ways for their implementation into the control structure. Section IV provides insight into the control mechanisms for the arm-sum energy control for both inverter and rectifier mode of operation. Section $\mathrm{V}$ provides evaluation of the analyzed control concepts, whereas the conclusions of this work are provided in Section VI.

\section{Direct Arm Energy Control}

\section{A. Motivation}

The goal of the arm energy control is to ensure that the energy content within each arm corresponds to the reference value. In normal operation, the number of SMs per arm is equal in all converter arms, and sum of their voltages is kept around the rated DC voltage, $V_{\mathrm{DC}}^{\mathrm{nom}}$. However, in case of failure of one or several SMs within an arm, remaining SMs are typically surcharged so that their sum voltage remains $V_{\mathrm{DC}}$, as during the normal operation [22]. In this case, the arm with failed SM contains more energy stored compared to the other arms. Therefore, each arm should be equipped with a dedicated energy controller in order to ensure direct control of their energy content.

Another reason why direct arm energy control is analysed is to explore energy control concepts that are not only applicable to a $3 \mathrm{PH}$ MMC, but also to other variations of an MMC, where arms are arranged in a different way. Therefore, there is a need for a generalized approach in control of arm energies.

In general, motivation for the energy control is not solely related the the previously mentioned fault case, but also to the imbalance between the arm energies that occurs during the transients, grid unbalances, or due to effects caused by inductance tolerances. Finally, using dedicated energy

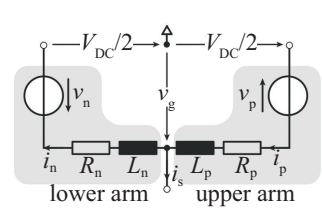

(a)

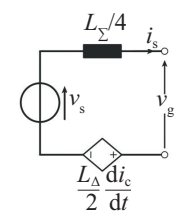

(b)

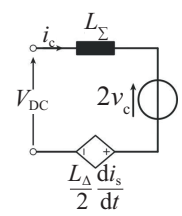

(c)
Fig. 1. Per-phase equivalent circuits of an MMC. (a) Equivalent circuit of an MMC phase-leg. (b) Arm-differential current equivalent circuit. (c) Armcommon current equivalent circuit.

controllers in order to gain asymptotic stability of the closedloop controlled MMC was suggested in [9].

As an example, a 3PH MMC is used to develop the direct arm energy control, which is performed through the socalled arm-differential energy control and arm-sum energy control. The former control mechanism ensures that the energy difference between the upper (p) and lower (n) arm (cf., Fig. 1(a)) of a single phase-leg $W_{\Delta}=W_{\mathrm{p}}-W_{\mathrm{n}}$ matches the difference between the arm energy references $W_{\Delta}^{*}=W_{\mathrm{p}}^{*}-W_{\mathrm{n}}^{*}$. The latter control mech-anism ensures that the sum energy of the belonging arms $W_{\Sigma}=W_{\mathrm{p}}+W_{\mathrm{n}}$ matches the sum of the arm energy references $W_{\Sigma}^{*}=W_{\mathrm{p}}^{*}+W_{\mathrm{n}}^{*}$. Consequently, with two energy controllers per phase-leg, energy content within each arm is controlled directly and independently.

\section{B. Control of the Arm-Energies}

Arm power equations, used for the purpose of the direct arm energy control, can be derived by referring to the equivalent circuit of a single phase-leg of an MMC (cf., Fig. 1(a)). Arm currents and voltages $\left(i_{\mathrm{p}}, i_{\mathrm{n}}, v_{\mathrm{p}}\right.$ and $\left.v_{\mathrm{n}}\right)$ can be expressed through their common $\left(i_{\mathrm{c}}, v_{\mathrm{c}}\right)$ and differential components $\left(i_{\mathrm{s}}, v_{\mathrm{s}}\right)$, defined by the expressions (1)-(4). Consequently, arm-power equations for the upper (p) and lower (n) arms take the form as in (5)-(6).

$$
\begin{gathered}
i_{\mathrm{c}}=\left(i_{\mathrm{p}}+i_{\mathrm{n}}\right) / 2 \\
v_{\mathrm{c}}=\left(v_{\mathrm{p}}+v_{\mathrm{n}}\right) / 2 \\
i_{\mathrm{s}}=i_{\mathrm{p}}-i_{\mathrm{n}} \\
v_{\mathrm{s}}=\left(v_{\mathrm{n}}-v_{\mathrm{p}}\right) / 2
\end{gathered}
$$

Based on (5)-(6) the expressions for the arm-differential power $p_{\Delta}$ and arm-sum power $p_{\Sigma}$ can be derived, as given in (7)-(8).

$$
\begin{aligned}
& p_{\mathrm{p}}=v_{\mathrm{p}} i_{\mathrm{p}}=\left(v_{\mathrm{c}}-v_{\mathrm{s}}\right)\left(i_{\mathrm{c}}+i_{\mathrm{s}} / 2\right) \\
& p_{\mathrm{n}}=v_{\mathrm{n}} i_{\mathrm{n}}=\left(v_{\mathrm{c}}+v_{\mathrm{s}}\right)\left(i_{\mathrm{c}}-i_{\mathrm{s}} / 2\right)
\end{aligned}
$$

Those two power components are control variables being in charge of the arm-differential $W_{\Delta}$ and arm-sum $W_{\Sigma}$ energy control, respectively. One can notice from (7)-(8) that it is necessary to have control over the arm-common and armdifferential voltages and currents in order to perform arm energy control.

$$
p_{\Delta}=p_{\mathrm{p}}-p_{\mathrm{n}}=v_{\mathrm{c}} i_{\mathrm{s}}-2 v_{\mathrm{s}} i_{\mathrm{c}}
$$




$$
p_{\Sigma}=p_{\mathrm{p}}+p_{\mathrm{n}}=2 v_{\mathrm{c}} i_{\mathrm{c}}-v_{\mathrm{s}} i_{\mathrm{s}}
$$

\section{MMC Decoupled Control and Energy Imbalances}

To determine the nature of these voltages and currents, applying the Kirchhoff's voltage law to the upper and lower arm (cf., Fig. 1(a)), the following equations are yielded:

$$
\begin{aligned}
& v_{\mathrm{g}}=\frac{V_{\mathrm{DC}}}{2}-v_{\mathrm{p}}-L_{\mathrm{p}} \frac{\mathrm{d} i_{\mathrm{p}}}{\mathrm{d} t} \\
& v_{\mathrm{g}}=-\frac{V_{\mathrm{DC}}}{2}+v_{\mathrm{n}}+L_{\mathrm{n}} \frac{\mathrm{d} i_{\mathrm{n}}}{\mathrm{d} t}
\end{aligned}
$$

For the sake of simplification, arm resistances are neglected in (9)-(10). Summing and subtracting (9)-(10), and introducing $L_{\Sigma}=L_{\mathrm{p}}+L_{\mathrm{n}}$ and $L_{\Delta}=L_{\mathrm{p}}-L_{\mathrm{n}}$ yields the equations for the equivalent loops of the arm-common $i_{\mathrm{c}}$ and arm-differential current $i_{\mathrm{s}}(11)-(12)$.

$$
\begin{gathered}
\frac{L_{\Sigma}}{4} \frac{\mathrm{d} i_{\mathrm{s}}}{\mathrm{d} t}=v_{\mathrm{s}}-v_{\mathrm{g}}-\frac{L_{\Delta}}{2} \frac{\mathrm{d} i_{\mathrm{c}}}{\mathrm{d} t} \\
L_{\Sigma} \frac{\mathrm{d} i_{\mathrm{c}}}{\mathrm{d} t}=V_{\mathrm{DC}}-2 v_{\mathrm{c}}-\frac{L_{\Delta}}{2} \frac{\mathrm{d} i_{\mathrm{s}}}{\mathrm{d} t}
\end{gathered}
$$

From the two figures (cf., Fig. 1(b) and (c)) one can notice that the arm-differential voltage $v_{\mathrm{s}}$ has an $\mathrm{AC}$ nature, and is in charge of the arm-differential current (grid current) control. The arm-common voltage $v_{\mathrm{c}}$ has a DC nature, and is in charge of the arm-common current $i_{\mathrm{c}}$ control. This current component consists of the DC part, which ideally corresponds to $i_{\mathrm{DC}} / 3$ in a $3 \mathrm{PH} \mathrm{MMC}$, and additional circulating current components, which circulate among the MMC legs, and remain unobserved at the MMC terminals. From (11)-(12) one can conclude that the arm-common current control and arm-differential current control on a per-phase level are decoupled from each other, provided that the upper and lower arm inductances match, i.e., $L_{\Delta}=0$. However, in practice this cannot truly be guaranteed due to manufacturing tolerances, and cross-coupling between the two control loops appears, as illustrated in Fig. 1(b) and (c). This results in parasitic AC current components in the armcommon current $i_{\mathrm{c}}$ and DC current component in the armdifferential current $i_{\mathrm{s}}$, which can cause arm-differential energy deviation.

\section{Arm-Differential Energy CONTrol}

Arm-differential energy control is based on the principles from (7). In terms of control, it is based on a feedback $P$ controllers (cf., Fig. 2), yielding necessary power references $P_{\Delta}^{*}$ per phase, which are further transformed to the appropriate arm-common current references $i_{\mathrm{c} \Delta}^{*}$. Both energy control actions (arm-differential and arm-sum) are internal control actions, and as such should not be observed either from the $\mathrm{AC}$, or from the DC terminals of an MMC. Therefore, current components generated for the purpose of energy control have to remain unobserved at both converter terminals.

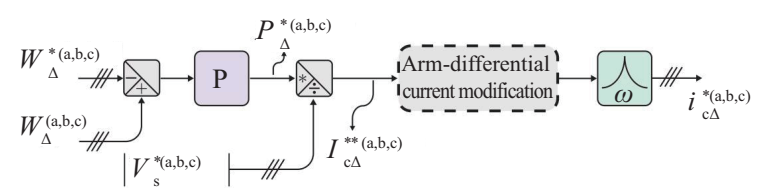

Fig. 2. Control structure of the arm-differential energy control.

\section{A. Arm-Differential Energy Control Mechanism}

Analyzing the power terms in (7), one can conclude that the average value of the arm-differential power $p_{\Delta}$ over a fundamental period has a non-zero value in case when a fundamental frequency component exists in the arm-common current $i_{\mathrm{c}}$. Therefore, arm-differential energy $W_{\Delta}$ can be controlled by introducing such current component $i_{\mathrm{c} \Delta}$. This current component should have circulating nature, i.e., remain unobserved at the converter terminals, and those currents are mostly used for the purpose of energy balancing [8], or to reduce voltage fluctuations within the SM capacitors [23], [24].

The average arm-differential power over a fundamental period of the phase voltage $v_{\mathrm{s}}$ is given by (13), where $V_{\mathrm{s}}$ denotes magnitude of the phase voltage, $I_{\mathrm{c} \Delta}$ denotes magnitude of the introduced fundamental frequency arm-common current component, whereas $\varphi$ denotes respective phase disposition among the two variables. Arm-differential voltage $v_{\mathrm{s}}$ is determined by the grid conditions, and is in charge of the grid (arm-differential) current $i_{\mathrm{s}}$ control.

$$
P_{\Delta}=V_{\mathrm{s}} I_{\mathrm{c} \Delta} \cos (\varphi)
$$

In order to realize arm-differential power reference with the lowest possible magnitude of the fundamental frequency armcommon current component $I_{\mathrm{c} \Delta}$, voltage and current should be in phase. However, due to the arbitrary arm-differential energy references among the phase-legs $W_{\Delta}^{*}$, obtained arm-common current references $i_{\mathrm{c} \Delta}^{* *}$ would have different magnitudes, and thus have non-zero sum among the phase-legs. Sum of those AC currents would appear in the DC link current, which should be avoided, so the fundamental frequency arm-common current references $i_{\mathrm{c} \Delta}^{* *}$ should be modified in order to have circulating nature.

\section{B. Arm-Differential Current Modification}

Fig. 3 illustrates the purpose of the arm-differential current modification block. Fig. 3(a) shows the original set of reference current vectors, obtained from the energy controllers (cf., Fig. 2). Reference current vectors $\vec{I}_{\mathrm{c} \Delta, x}^{* *}$ do not generally sum-up to zero, and would appear in the DC link current, which justifies the need for their modification.

One way of modifying the set of original current vectors $\vec{I}_{\mathrm{c} \Delta}^{* *}$ is shown in Fig. 3(b). Projections of the modified set of current vectors $\vec{I}_{\mathrm{c} \Delta}^{*}$ to their associated phase-voltage vectors match the original current references. Therefore, proposed transformation ensures preservation of active powers, being the outputs of arm-differential energy controllers from Fig. 2. Moreover, 


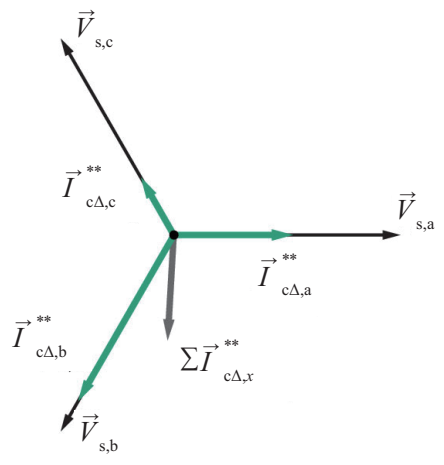

(a)

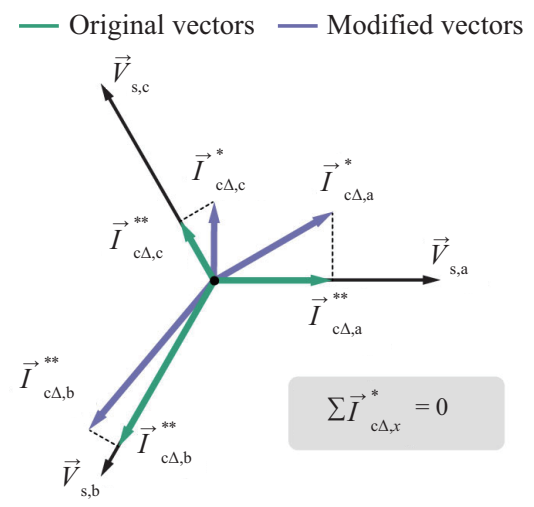

(b)

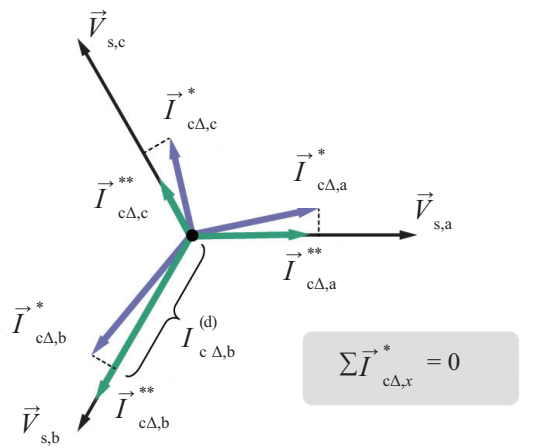

(c)

Fig. 3. Modifications of the original current vectors within arm-differential current modification block. (a) Original set of current vectors. (b) Current modification with method I. (c) Current modification with method II.

sum of newly obtained current vectors equals zero, which is a control goal determined during the previous discussion. This method of current vectors modification will be further on referred to as "method I".

Another approach is presented in Fig. 3(c), where the modified set of vectors $\vec{I}_{\mathrm{c} \Delta}^{*}$ again sum-up to zero, however their projections to the corresponding phase-voltage axes do not match the original vectors. Consequently, power preservation is not guaranteed. Henceforth, this method will be referred to as "method II".

What the two methods have in common is that the modified set of vectors sum-up to zero, which can be expressed as in (14). Throughout the paper, 3PH MMC will be used as a showcase, without loss of generality. Magnitudes of the modified set of vectors are denoted as $I_{\mathrm{c} \triangle x}^{*}$, whereas their phase shifts with respect to the corresponding phase-voltages are denoted by $\varphi_{x}$.

$$
\begin{aligned}
I_{\mathrm{c} \triangle \mathrm{a}, \mathrm{c}}^{*} \cos \left(\omega t-\varphi_{\mathrm{a}}\right) & +I_{\mathrm{c} \triangle \mathrm{b}}^{*} \cos \left(\omega t-2 \pi / 3-\varphi_{\mathrm{b}}\right) \\
& +I_{\mathrm{c} \Delta \mathrm{c}, \mathrm{c}}^{*} \cos \left(\omega t+2 \pi / 3-\varphi_{\mathrm{c}}\right)=0
\end{aligned}
$$

Introducing the projections of the modified set of current vectors to the corresponding phase-voltage axes as $I_{\mathrm{c} \Delta, x}^{(\mathrm{d})}=$ $I_{\mathrm{c} \triangle, x}^{*} \cos \left(\varphi_{x}\right)$, condition (14) can be decoupled into the two independent conditions, expressed in (15)-(16).

$$
\begin{aligned}
& \sqrt{3} I_{\mathrm{c} \Delta, \mathrm{b}}^{(\mathrm{d})} \tan \left(\varphi_{\mathrm{b}}\right)=I_{\mathrm{c} \Delta, \mathrm{a}}^{(\mathrm{d})}\left[1+\sqrt{3} \tan \left(\varphi_{\mathrm{a}}\right)\right]+I_{\mathrm{c} \Delta, \mathrm{b}}^{(\mathrm{d})}-2 I_{\mathrm{c} \Delta, \mathrm{c}}^{(\mathrm{d})}(1 \\
& \sqrt{3} I_{\mathrm{c} \Delta, \mathrm{c}}^{(\mathrm{d})} \tan \left(\varphi_{\mathrm{c}}\right)=I_{\mathrm{c} \Delta, \mathrm{a}}^{(\mathrm{d})}\left[\sqrt{3} \tan \left(\varphi_{\mathrm{a}}\right)-1\right]+2 I_{\mathrm{c} \Delta, \mathrm{b}}^{(\mathrm{d})}-I_{\mathrm{c} \Delta, \mathrm{c}}^{(\mathrm{d})}(1)
\end{aligned}
$$

Modified set of current vectors consists of three current vectors, with each one of them being defined by its magnitude $I_{c \triangle x}^{*}$ and phase $\varphi_{x}$, giving in total six variables that could generally be selected arbitrarily. However, regardless of the chosen method, conditions given by (15)-(16) reduce the degree of freedom from six to four. Remaining degrees of freedom (three vector projections $I_{\mathrm{c} \Delta, x}^{(\mathrm{d})}$ and $\tan \left(\varphi_{\mathrm{a}}\right)$ in (15)-(16)) can be used in different ways, as will be demonstrated shortly.

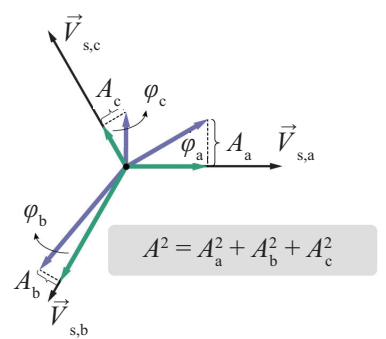

(a)

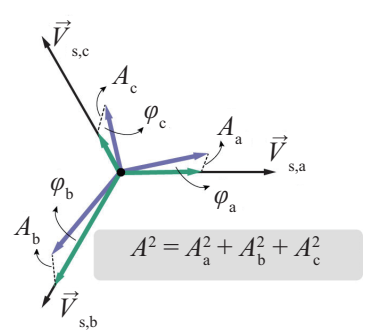

(b)
Fig. 4. Arm-differerntial current modification with corresponding criterion function. (a) method I and (b) method II (green—original vectors; purple — modified vectors).

\section{Method I}

Method I ensures that the projections of the modified current vectors to the corresponding phase-voltage axes match the current references obtained from the energy controllers $I_{\mathrm{c} \Delta, x}^{(\mathrm{d})}$ $=I_{\mathrm{c} \Delta, x}^{* *}$. The last condition applied to all three phase-legs of an MMC, further reduces the degree of freedom in choosing the modified set of vectors from four to one. The only variable that can be arbitrarily chosen in (15)-(16) is $\tan \left(\varphi_{\mathrm{a}}\right)$, and there are numerous ways of choosing the variable value.

Since the original set of current references $I_{\mathrm{c} \Delta, x}^{* *}$ have the lowest magnitudes for a given power reference $P_{\Delta}^{*}$, modified set of current vectors should have minimum deviation from the original set of vectors, in order to minimize the current stress imposed on the converter. The deviation function $A^{2}$ is introduced (cf., Fig. 4(a)), and can be expressed as in (17).

$$
\begin{aligned}
A^{2} & =A_{\mathrm{a}}^{2}+A_{\mathrm{b}}^{2}+A_{\mathrm{c}}^{2} \\
& =\left[I_{\mathrm{c} \Delta, \mathrm{a}}^{* *} \tan \left(\varphi_{\mathrm{a}}\right)\right]^{2}+\left[I_{\mathrm{c} \Delta, \mathrm{b}}^{* *} \tan \left(\varphi_{\mathrm{b}}\right)\right]^{2}+\left[I_{\mathrm{c} \Delta, \mathrm{c}}^{* *} \tan \left(\varphi_{\mathrm{c}}\right)\right]^{2}
\end{aligned}
$$

Incorporating (15)-(16) into (17) and minimizing the resulting function of a single variable $\tan \left(\varphi_{\mathrm{a}}\right)$ (it could have been any other phase taken as a reference - the result would be the same), yields the optimal angle $\tan \left(\varphi_{\mathrm{a}}\right)$, and thus all the other 
parameters of the modified set of vectors:

$$
\begin{aligned}
& \tan \left(\varphi_{\mathrm{a}}\right)=\frac{I_{\mathrm{c} \Delta, \mathrm{c}}^{* *}-I_{\mathrm{c} \Delta, \mathrm{b}}^{* *}}{\sqrt{3} I_{\mathrm{c} \Delta, \mathrm{a}}^{* *}} \\
& \tan \left(\varphi_{\mathrm{b}}\right)=\frac{I_{\mathrm{c} \Delta, \mathrm{a}}^{* *}-I_{\mathrm{c} \Delta, \mathrm{c}}^{* *}}{\sqrt{3} I_{\mathrm{c} \Delta, \mathrm{b}}^{* *}} \\
& \tan \left(\varphi_{\mathrm{c}}\right)=\frac{I_{\mathrm{c} \Delta, \mathrm{b}}^{* *}-I_{\mathrm{c} \Delta, \mathrm{a}}^{* *}}{\sqrt{3} I_{\mathrm{c} \Delta, \mathrm{c}}^{* *}} .
\end{aligned}
$$

Finally, time-domain expressions for the modified set of currents are as follows:

$$
\begin{aligned}
i_{\mathrm{c} \Delta \mathrm{a}}^{*}(t)= & I_{\mathrm{c} \Delta, \mathrm{a}} \cos \left(\omega t-\varphi_{\mathrm{a}}\right) \\
= & I_{\mathrm{c} \Delta, \mathrm{a}}^{(\mathrm{d})} \cos (\omega t)+I_{\mathrm{c} \Delta, \mathrm{a}}^{(\mathrm{d})} \sin (\omega t) \tan \left(\varphi_{\mathrm{a}}\right) \\
= & I_{\mathrm{c} \Delta, \mathrm{a}}^{* *} \cos (\omega t)+\frac{1}{\sqrt{3}}\left(I_{\mathrm{c} \Delta, \mathrm{c}}^{* *}-I_{\mathrm{c} \Delta, \mathrm{b}}^{* *}\right) \sin (\omega t) \\
i_{\mathrm{c} \Delta, \mathrm{b}}^{*}(t)= & I_{\mathrm{c} \Delta, \mathrm{b}}^{* *} \cos \left(\omega t-\frac{2 \pi}{3}\right) \\
& +\frac{1}{\sqrt{3}}\left(I_{\mathrm{c} \Delta, \mathrm{a}}^{* *}-I_{\mathrm{c} \Delta, \mathrm{c}}^{* *}\right) \sin \left(\omega t-\frac{2 \pi}{3}\right) \\
i_{\mathrm{c} \Delta, \mathrm{c}}^{*}(t)= & I_{\mathrm{c} \Delta, \mathrm{c}}^{* *} \cos \left(\omega t+\frac{2 \pi}{3}\right) \\
& +\frac{1}{\sqrt{3}}\left(I_{\mathrm{c} \Delta, \mathrm{b}}^{* *}-I_{\mathrm{c} \Delta, \mathrm{a}}^{* *}\right) \sin \left(\omega t+\frac{2 \pi}{3}\right)
\end{aligned}
$$

Regarding the implementation of this method of current modification, generation of the instantaneous values of modified current references can be done in a simple manner, using readily available signals. Namely, should one denote normalized arm-differential voltage references as $A=\cos (\omega t)$, $B=\cos (\omega t-2 \pi / 3)$ and $C=\cos (\omega t+2 \pi / 3),(21)-(23)$ can be rewritten in a way presented in (24)-(26).

$$
\begin{aligned}
& \dot{i}_{\mathrm{c} \Delta, \mathrm{a}}^{*}(t)=I_{\mathrm{c} \Delta, \mathrm{a}}^{* *} A+\frac{1}{3}\left(I_{\mathrm{c} \Delta, \mathrm{c}}^{* *}-I_{\mathrm{c} \Delta, \mathrm{b}}^{* *}\right)(B-C) \\
& i_{\mathrm{c} \Delta, \mathrm{b}}^{*}(t)=I_{\mathrm{c} \Delta, \mathrm{b}}^{* *} B+\frac{1}{3}\left(I_{\mathrm{c} \Delta, \mathrm{a}}^{* *}-I_{\mathrm{c} \Delta, \mathrm{c}}^{* *}\right)(C-A) \\
& i_{\mathrm{c} \Delta, \mathrm{c}}^{*}(t)=I_{\mathrm{c} \Delta, \mathrm{c}}^{* *} C+\frac{1}{3}\left(I_{\mathrm{c} \Delta, \mathrm{b}}^{* *}-I_{\mathrm{c} \Delta, \mathrm{a}}^{* *}\right)(A-B)
\end{aligned}
$$

Modified set of current references (21)-(23) corresponds to the one presented in [15], although the process of derivation and arm-differential current modification block implementation are completely different from [15]. [15] proposes decomposition of the reference current vectors into the positive and negative sequence, due to asymmetry of generated current components. This paper, on the other hand, offers an easy way for the circulating current reference generation using readily available signals (cf., Fig. 5(a)), without the need for positive/ negative sequence decomposition.

Comparison of current references obtained in a way proposed by [15] and this paper, yields the same results. As an

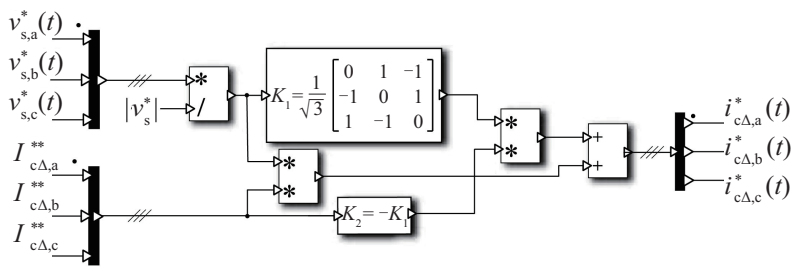

(a)

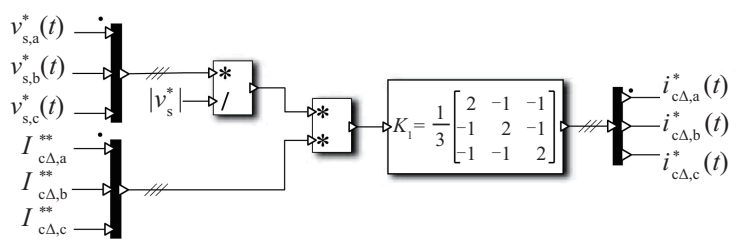

(b)

Fig. 5. Implementation of arm-differential current modification block in case of (a) method I and (b) method II.

TABLE I

Test Converter Parameters

\begin{tabular}{lcc}
\hline \hline Parameter & Label & Value \\
\hline Line voltage & $U_{\mathrm{n}}$ & $3.3 \mathrm{kV}$ \\
Apparent power & $S_{\mathrm{n}}$ & $250 \mathrm{kVA}$ \\
Grid frequency & $f_{\mathrm{n}}$ & $50 \mathrm{~Hz}$ \\
Number of SMs per arm & $N_{\mathrm{SM}}$ & 8 \\
SM capacitance (tolerance $\pm 5 \%)$ & $C_{\mathrm{SM}}$ & $2.25 \mathrm{mF}$ \\
Arm inductance & $L_{\mathrm{br}}$ & $2.5 \mathrm{mH}$ \\
Arm resistance & $R_{\mathrm{br}}$ & $20 \mathrm{~m} \Omega$ \\
Switching frequency & $f_{\mathrm{sw}}$ & $1000 \mathrm{~Hz}$ \\
\hline \hline
\end{tabular}

example, assuming the original current references being $I_{\mathrm{c} \triangle \mathrm{a}, \mathrm{a}}^{* *}=$ $0.6 \angle 0^{\circ}$ p.u., $I_{\mathrm{c} \Delta, \mathrm{b}}^{* *}=0.2 \angle-120^{\circ}$ p.u., $I_{\mathrm{c} \Delta, \mathrm{c}}^{* *}=0.8 \angle 120^{\circ}$ p.u., modified set of vectors in both cases takes the following form: $I_{\mathrm{c} \triangle, \mathrm{a}}^{* *}=0.6928 \angle-30^{\circ}$ p.u., $I_{\mathrm{c} \Delta, \mathrm{b}}^{* *}=0.2309 \angle-90^{\circ}$ p.u., $I_{\mathrm{c} \Delta, \mathrm{c}}^{* *}=$ $0.8327 \angle 136.1^{\circ}$ p.u..

$3 \mathrm{PH}$ MMC, with the parameters provided in Table I, is simulated in PLECS. At the time instant $t=0.6 \mathrm{~s}$, references for the two arm energies $W_{x, y}^{*}$ are changed, and returned to the nominal values at $t=0.9 \mathrm{~s}$. As a result, references for the arm-differential $W_{\Delta, x}^{*}$ and arm-sum energies $W_{\Sigma, x}^{*}$ are changed accordingly. Fig. 6 shows the response of the arm-differential energy controller, together with its arm-common current references $i_{\mathrm{c} \Delta, x}^{*}$, representing modified current references implemented using method I. Settling time for the armdifferential energies is approximately $50 \mathrm{~ms}$.

\section{Method II}

Projections of the modified current vectors to the corresponding axes do not necessarily need to match the reference values, as it was the case with the previous method. This way of vectors modification is depicted in Figs. 3(c) and 4(b), and it is being referred to as method II in this paper. Since the 

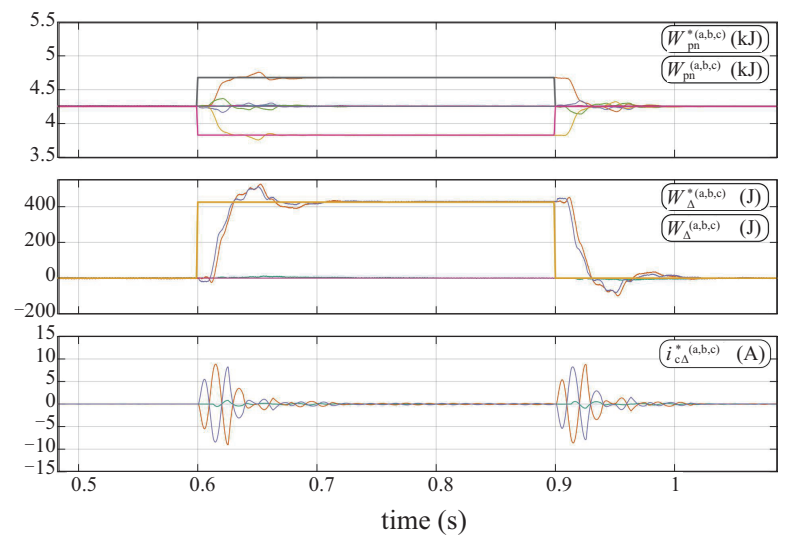

Fig. 6. Arm-differential energy control performance after a step change in armenergies references and arm-differential current modification performed with method I.

parameters of the modified set of vectors are now constrained only by the conditions given in $(15)-(16)$, there are four degrees of freedom left in choosing their values.

For the same reason as in the previous method, it is desirable to find the modified set of vectors with the minimum deviation from the original one, under the constraints (15)-(16). Additional degrees of freedom can be leveraged to obtain a set of current vectors that has lower deviation from the original one, compared to the method I, thus further reducing current stress on the converter. As in the previous case, the deviation function $A^{2}$ is created (cf., Fig. 4(b)) and it can be expressed as in (27). This equation has six variables, however taking into account constraints imposed by (15)-(16), the number of independent variables is four, i.e., $A=f\left(I_{\mathrm{c} \Delta, \mathrm{a},}^{(\mathrm{d})}, I_{\mathrm{c} \Delta, \mathrm{b}}^{(\mathrm{d})}, I_{\mathrm{c} \Delta, \mathrm{c}}^{(\mathrm{d})}, \tan \left(\varphi_{\mathrm{a}}\right)\right)$.

$$
\begin{aligned}
A^{2}= & \left(I_{\mathrm{c} \Delta, \mathrm{a}}^{* *}-I_{\mathrm{c} \Delta, \mathrm{a}}^{(\mathrm{d})}\right)^{2}+\left[I_{\mathrm{c} \Delta, \mathrm{a}}^{(\mathrm{d})} \tan \left(\varphi_{\mathrm{a}}\right)\right]^{2} \\
& +\left(I_{\mathrm{c} \Delta, \mathrm{b}}^{* *}-I_{\mathrm{c} \Delta, \mathrm{b}}^{(\mathrm{d})}\right)^{2}+\left[I_{\mathrm{c} \Delta, \mathrm{b}}^{(\mathrm{d})} \tan \left(\varphi_{\mathrm{b}}\right)\right]^{2} \\
& +\left(I_{\mathrm{c} \Delta, \mathrm{c}}^{* *}-I_{\mathrm{c} \Delta, \mathrm{c}}^{(\mathrm{d})}\right)^{2}+\left[I_{\mathrm{c} \Delta, \mathrm{c}}^{(\mathrm{d})} \tan \left(\varphi_{\mathrm{c}}\right)\right]^{2}
\end{aligned}
$$

Minimization of (27), yields the desired set of parameters, given by (28)-(29). Consequently, time-domain expressions for the modified set of current vectors take the form as in (30)-(32).

$$
\begin{aligned}
& \left\{\begin{array}{l}
I_{\mathrm{c} \Delta, \mathrm{a}}^{(\mathrm{d})}=\frac{4 I_{\mathrm{c} \Delta, \mathrm{a}}^{* *}+I_{\mathrm{c} \Delta, \mathrm{b}}^{* *}+I_{\mathrm{c} \Delta, \mathrm{c}}^{* *}}{6} \\
I_{\mathrm{c} \Delta, \mathrm{b}}^{(\mathrm{d})}=\frac{I_{\mathrm{c} \Delta, \mathrm{a}}^{* *}+4 I_{\mathrm{c} \Delta, \mathrm{b}}^{* *}+I_{\mathrm{c} \Delta, \mathrm{c}}^{* *}}{6} \\
I_{\mathrm{c} \Delta, \mathrm{c}}^{(\mathrm{d})}=\frac{I_{\mathrm{c} \Delta, \mathrm{a}}^{* *}+I_{\mathrm{c} \Delta, \mathrm{b}}^{* *}+4 I_{\mathrm{c} \Delta, \mathrm{c}}^{* *}}{6} \\
\tan \left(\varphi_{\mathrm{a}}\right)=\frac{\sqrt{3}\left(I_{\mathrm{c} \Delta, \mathrm{c}}^{* *}-I_{\mathrm{c} \Delta, \mathrm{b}}^{* *}\right)}{4 I_{\mathrm{c} \Delta, \mathrm{a}}^{* *}+I_{\mathrm{c} \Delta, \mathrm{b}}^{* *}+I_{\mathrm{c} \Delta, \mathrm{c}}^{* *}} \\
\tan \left(\varphi_{\mathrm{b}}\right)=\frac{\sqrt{3}\left(I_{\mathrm{c} \Delta, \mathrm{a}}^{* *}-I_{\mathrm{c} \Delta, \mathrm{c}}^{* *}\right)}{I_{\mathrm{c} \Delta, \mathrm{a}}^{* *}+4 I_{\mathrm{c} \Delta, \mathrm{b}}^{* *}+I_{\mathrm{c} \Delta, \mathrm{c}}^{* *}} \\
\tan \left(\varphi_{\mathrm{c}}\right)=\frac{\sqrt{3}\left(I_{\mathrm{c} \Delta, \mathrm{b}}^{* *}-I_{\mathrm{c} \Delta, \mathrm{a}}^{* *}\right)}{I_{\mathrm{c} \Delta, \mathrm{a}}^{* *}+I_{\mathrm{c} \Delta, \mathrm{b}}^{* *}+4 I_{\mathrm{c} \Delta, \mathrm{c}}^{* *}}
\end{array}\right.
\end{aligned}
$$

Analyzing the resulting time-domain expressions for the modified arm-current references (30)-(32), it can be observed that they correspond to the original current references reduced by their average (zero) value. Implementation of armdifferential current modification block is depicted in Fig. 5(b). Although represented by multiplication with the matrix $K_{1}$, modified current references $i_{\mathrm{c} \Delta, x}^{*}$ are obtained simply by subtracting the average value of all three current references from the corresponding original current reference.

This result could have also been obtained by analysing the space-vector of the original set of references. In general, there could be $n$ phases, where the equivalent space-vector can be represented by any set of $n$ linearly-independent vectors, with one of them being zero (average) vector. In that case, the closest approximation of the original set of vectors, under the constraints that the modified vectors should sum-up to zero, is yielded by a simple subtraction of the average (zero) component from the original space-vector. The resulting spacevector preserves its projections to the remaining axes and thus represents the best possible approximation of the original space-vector. An example of these theoretical considerations on a $3 \mathrm{PH}$ basis is illustrated in Fig. 7, where the original set of three current references is transformed to the space-vector in $\alpha \beta 0$ reference frame. Modified space-vector $\vec{I}^{*}$ in $\alpha \beta 0$ is

$$
\begin{aligned}
i_{\mathrm{c} \Delta, \mathrm{a}}^{*}(t) & =I_{\mathrm{c} \Delta, \mathrm{a}} \cos \left(\omega t-\varphi_{\mathrm{a}}\right)=I_{\mathrm{c} \Delta, \mathrm{a}}^{(\mathrm{d})} \cos (\omega t)+I_{\mathrm{c} \Delta, \mathrm{a}}^{(\mathrm{d})} \sin (\omega t) \tan \left(\varphi_{\mathrm{a}}\right) \\
& =\frac{2}{3} I_{\mathrm{c} \Delta, \mathrm{a}}^{* *} \cos (\omega t)-\frac{1}{3} I_{\mathrm{c} \Delta, \mathrm{b}}^{* *} \cos \left(\omega t-\frac{2 \pi}{3}\right)-\frac{1}{3} I_{\mathrm{c} \Delta, \mathrm{c}}^{* *} \cos \left(\omega t+\frac{2 \pi}{3}\right) \\
& =I_{\mathrm{c} \Delta, \mathrm{a}}^{* *} A-\frac{1}{3}\left(I_{\mathrm{c} \Delta, \mathrm{a}}^{* *} A+I_{\mathrm{c} \Delta, \mathrm{b}}^{* *} B+I_{\mathrm{c} \Delta, \mathrm{c}}^{* *} C\right) \\
\dot{i}_{\mathrm{c} \Delta, \mathrm{b}}^{*}(t) & =-\frac{1}{3} I_{\mathrm{c} \Delta, \mathrm{a}}^{* *} \cos (\omega t)+\frac{2}{3} I_{\mathrm{c} \Delta, \mathrm{b}}^{* *} \cos \left(\omega t-\frac{2 \pi}{3}\right)-\frac{1}{3} I_{\mathrm{c} \Delta, \mathrm{c}}^{* *} \cos \left(\omega t+\frac{2 \pi}{3}\right) \\
& =I_{\mathrm{c} \Delta, \mathrm{b}}^{* *} B-\frac{1}{3}\left(I_{\mathrm{c} \Delta, \mathrm{a}}^{* *} A+I_{\mathrm{c} \Delta, \mathrm{b}}^{* *} B+I_{\mathrm{c} \Delta, \mathrm{c}}^{* *} C\right) \\
i_{\mathrm{c} \Delta, \mathrm{c}}^{*}(t) & =-\frac{1}{3} I_{\mathrm{c} \Delta, \mathrm{a}}^{* *} \cos (\omega t)-\frac{1}{3} I_{\mathrm{c} \Delta, \mathrm{b}}^{* *} \cos \left(\omega t-\frac{2 \pi}{3}\right)+\frac{2}{3} I_{\mathrm{c} \Delta, \mathrm{c}}^{* *} \cos \left(\omega t+\frac{2 \pi}{3}\right) \\
& =I_{\mathrm{c} \Delta, \mathrm{c}}^{* *} C-\frac{1}{3}\left(I_{\mathrm{c} \Delta, \mathrm{a}}^{* *} A+I_{\mathrm{c} \Delta, \mathrm{b}}^{* *} B+I_{\mathrm{c} \Delta, \mathrm{c}}^{* *} C\right)
\end{aligned}
$$




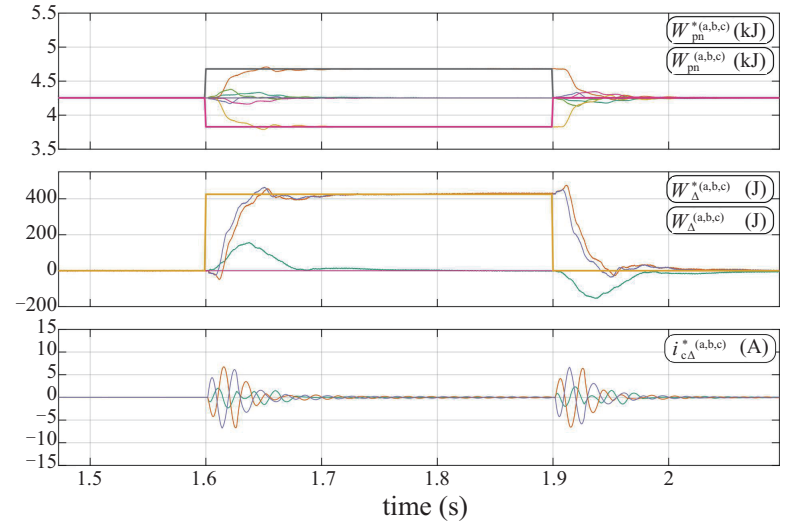

Fig. 7. Arm-differential energy control performance after a step change in arm-energies references and arm-differential current modification performed with method II.

obtained when the zero component $\vec{I}_{0}^{* *}$ is subtracted from the original space-vector $\vec{I}^{* *}$. Original $\alpha$ and $\beta$ components remain unaltered.

The result obtained here is generic, and can be applied to an MMC with arbitrary number of phases. Namely, in order to obtain arm-common current components with the lowest deviation from the original set of arm-common current components $i_{\mathrm{c} \Delta, x}^{* *}$, providing they sum-up to zero, the general approach can be described as:

$$
i_{\mathrm{c} \Delta, x}^{*}=i_{\mathrm{c} \Delta, x}^{* *}-\frac{1}{k} \sum_{y=1}^{k} i_{\mathrm{c} \Delta, y}^{* *},
$$

where the variable $k$ stands for the number of MMC phases. In addition to not being observed at the converter's terminals, thus modified currents pose less stress to the converter, compared to the ones obtained by the method I, due to the fact that their space-vector corresponds to the original space-vector reduced for its average (zero) component.

Reference [16] deals with the current vector modification in a generalized manner, and resulting transformation matrix is the same as in Fig. 5(b). However, interpretation of the results being presented in [16] might be tedious, and requires the use of advanced mathematical tools [21]. On the other hand, this paper introduces the problem, formulates the optimization criterion, and yields the solution in a simple manner, using simple mathematical manipulations. Moreover, the results are interpreted and clear guidelines for the method II application to an MMC with an arbitrary number of phases are provided.

To verify this approach, simulation used for method I verification is repeated, with the results being provided in Fig. 8. At the time instant $t=1.6 \mathrm{~s}$, references for the two arm energies are changed in the same fashion as previously, and so are the arm-differential energy references. As in the previous case, references are reached within $50 \mathrm{~ms}$. Difference between two methods of arm-differential current modification are in the fact that method II yields current references with lower magnitudes, yet it affects arm-common currents and arm-differential energies of all phase-legs within the converter. In contrast,

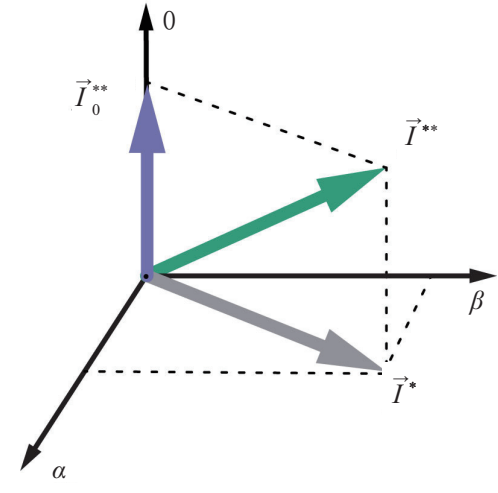

Fig. 8. Graphical explanation of the effects imposed by method II to the original space-vector.

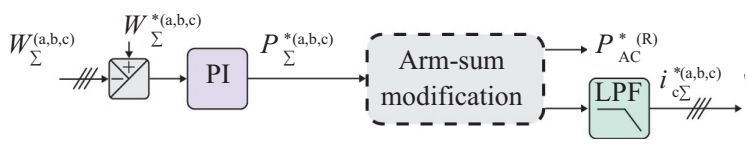

Fig. 9. Control structure of the arm-sum energy control.

method I only affects phase-legs where the control action is requested. Additionally, method II can be easily applied where number of phases is greater than three, and is more suitable for unbalanced grid conditions.

\section{Arm-Sum Energy Control}

The other energy control mechanism aims to control the arm-sum energy within a phase-leg, and together with the armdifferential energy control performs direct arm energy control. In order to identify mechanisms of control, one should refer to (34), derived from (8).

$$
p_{\Sigma}=2 v_{\mathrm{c}} i_{\mathrm{c}}-v_{\mathrm{s}} i_{\mathrm{s}}
$$

The first integrand term is a product of the arm-common voltage and the arm-common current. The arm-common voltage consists of its dominant DC part $V_{\mathrm{DC}} / 2$, and additional voltage component $\Delta v_{\mathrm{c}}$, used for the arm-common current $i_{\mathrm{c}}$ control. The arm-common current contains portion of the DC terminal current, ideally $i_{\mathrm{DC}} / 3$ in a $3 \mathrm{PH} \mathrm{MMC}$, and can also contain $\mathrm{AC}$ components for the purpose of arm-sum energy control, SM voltage ripple reduction, etc. Average value of the first integrand term in (34) over a fundamental period is equal to the DC power exchange of a single phase-leg with the DC link. Average value of the second product over a fundamental period is equal to the average active power exchange with a single phase of an AC system.

Control structure of arm-sum energy control is illustrated in Fig. 9, and it is based on a feedback PI controller, necessary during unbalanced grid conditions. Structure of arm-sum modification block differs slightly depending on the operation mode of an MMC (rectifier or inverter), and is presented in Fig. 10.

When MMC operates as an inverter, grid current is 


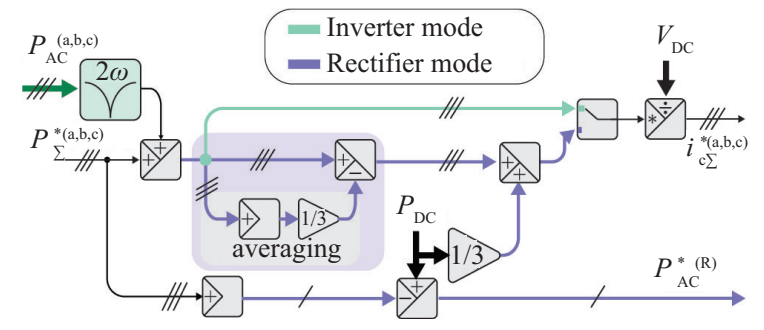

Fig. 10. Structure of the arm-sum modification block for both operating modesrectifier and inverter.

determined by the active and reactive power that $\mathrm{MMC}$ is supposed to provide to the grid. Therefore arm-sum energy control is performed with the arm-common currents $i_{\mathrm{c}}$. Output of arm-sum energy controllers $P_{\Sigma}^{*}$ are summed with the average value of the product $v_{\mathrm{s}} i_{\mathrm{s}}$, which is a single-phase active power delivered to the AC grid. Arm-common current reference is generated according to (35). Sum of the currents $i_{\mathrm{c} \Sigma}$ of all MMC phases is the DC terminal current.

$$
V_{\mathrm{DC}} i_{\mathrm{c} \Sigma}^{*}=v_{\mathrm{s}} i_{\mathrm{s}}+p_{\Sigma}^{*}
$$

In case when an MMC operates as a rectifier, it acts either as a DC current or voltage source. In this case, arm-sum controller power requirements $p_{\Sigma}^{*}$ are achieved by controlling the grid current $i_{\mathrm{s}}$, and consequently the product $v_{\mathrm{s}} i_{\mathrm{s}}$. Arbitrary power requirements $P_{\Sigma}^{*}$ among the phase-legs result in different requirements of the $v_{\mathrm{s}} i_{\mathrm{s}}$ product among the MMC phases. In both symmetrical and asymmetrical grid conditions, this would lead to the asymmetrical grid current $i_{\mathrm{s}}$ requirements, which is generally not permitted. Therefore, AC power reference $P_{\mathrm{AC}}^{*}{ }^{(\mathrm{R})}$ is obtained by summing (36) for all MMC phases (37).

$$
\begin{gathered}
p_{\Sigma}^{*}=V_{\mathrm{DC}} i_{\mathrm{c}}-v_{\mathrm{s}} i_{\mathrm{s}} \Rightarrow v_{\mathrm{s}} i_{\mathrm{s}}=V_{\mathrm{DC}} i_{\mathrm{c}}-p_{\Sigma}^{*} \\
P_{\mathrm{AC}}^{*}{ }^{(\mathrm{R})}=\sum_{x=\mathrm{a}, \mathrm{b}, \mathrm{c}} v_{\mathrm{s}}^{(x)} i_{\mathrm{s}}^{(x)}=V_{\mathrm{DC}} i_{\mathrm{DC}}-\sum_{x=\mathrm{a}, \mathrm{b}, \mathrm{c}} p_{\Sigma}^{*(x)}
\end{gathered}
$$

However, due to the fact that each phase-leg receives portion of the requested $\mathrm{AC}$ power $P_{\mathrm{AC}}^{*}$, neither does it correspond to the energy controller requests $P_{\Sigma}^{*}$ in each particular phase, nor is it equal among the phase-legs during grid unbalances. Therefore, initial assumption of equal arm-common currents among the three phase-legs $i_{\mathrm{c}}=i_{\mathrm{DC}} / 3$ has to be modified, by introducing the additional arm-common DC current components $\Delta i_{\mathrm{c} \Sigma}(38)$. Substituting (38) into (34) yields (39)-(40).

$$
\begin{gathered}
i_{\mathrm{c} \Sigma}=i_{\mathrm{DC}} / 3+\Delta i_{\mathrm{c} \Sigma} \\
p_{\Sigma}^{*}=V_{\mathrm{DC}}\left(i_{\mathrm{DC}} / 3+\Delta i_{\mathrm{c} \Sigma}\right)-v_{\mathrm{s}} i_{\mathrm{s}} \\
V_{\mathrm{DC}} \Delta i_{\mathrm{c} \Sigma}=-V_{\mathrm{DC}} i_{\mathrm{DC}} / 3+v_{\mathrm{s}} i_{\mathrm{s}}+p_{\Sigma}^{*}
\end{gathered}
$$

Sum of the $i_{\mathrm{c} \Sigma}$ currents among the three phase-legs has to remain equal to the $\mathrm{DC}$ current $i_{\mathrm{DC}}$, leading to the condition $\sum \Delta i_{\mathrm{c} \Sigma}=0$. These current components intend to cover the

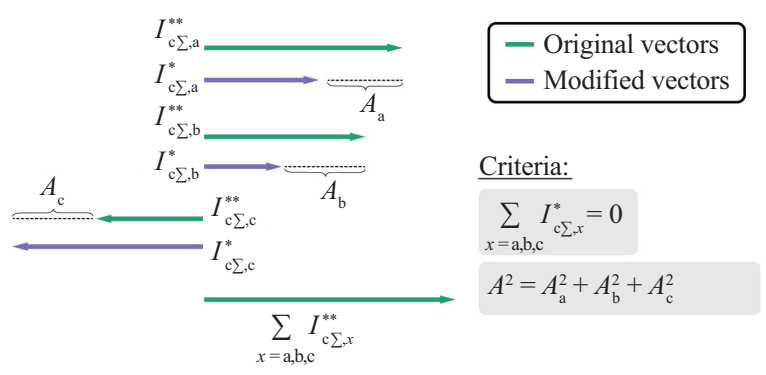

Fig. 11. Modification of the original set of arm-common currents in arm-sum energy control. Modified currents have DC nature.

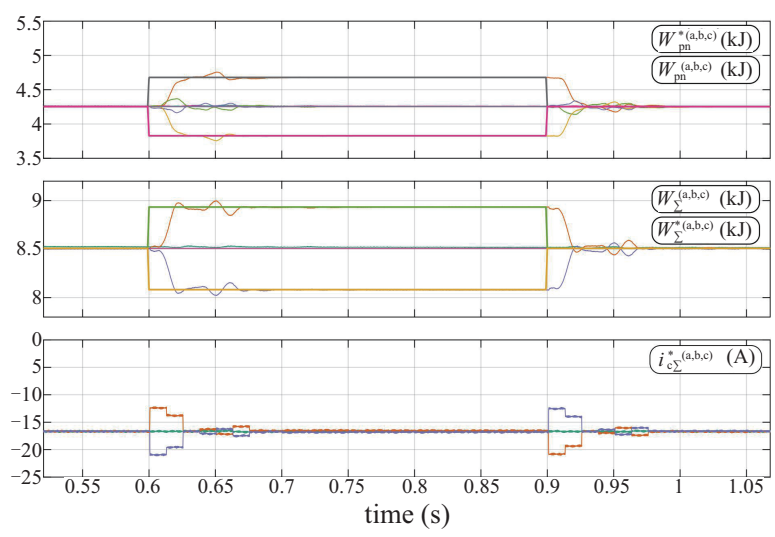

Fig. 12. Arm-sum energy control performance after a step change in armenergies references in inverter mode of operation.

difference between the DC power $\left(V_{\mathrm{DC}} i_{\mathrm{DC}} / 3\right)$ and actual $\mathrm{AC}$ power $\left(v_{\mathrm{s}} i_{\mathrm{s}}\right)$ per phase, as well as to satisfy arm-sum energy controller requirements $p_{\Sigma}^{*}$, as shown in (40).

Referring to Fig. 11, where a $3 \mathrm{PH}$ system is taken as an example, one can observe the original set of currents obtained from the arm-sum energy controllers $I_{\mathrm{c} \Sigma, x}^{* *}$, which do not sumup to zero. Therefore, as in the case of arm-differential energy control, those currents have to be modified in order to comply with the constraint. Since there are three currents, which have a DC nature, they are characterized only by their magnitudes. Therefore, the initial degree of freedom in choosing their modified values is three. However, taking into account the constraint that the modified set of currents should sum-up to zero, the degree of freedom is further reduced to two. It becomes obvious that the power references $p_{\Sigma, x}^{*}$ cannot be respected (as it was the case with method I of arm-differential energy control, or in the inverter mode of arm-sum energy control) without interfering with the DC terminal current, and a question how to use the remaining two degrees of freedom arises.

Again, the criterion function $A^{2}$ can be introduced (cf., Fig. 11), with the aim of minimizing the deviation of the modified set of currents with respect to the original one. The derivation is omitted for the sake of brevity, and only the final result is provided (41).

$$
i_{\mathrm{c} \sum, x}^{*}=i_{\mathrm{c} \sum, x}^{* *}-\frac{1}{3} \sum_{y=1}^{3} i_{\mathrm{c} \sum, y}^{* *}
$$




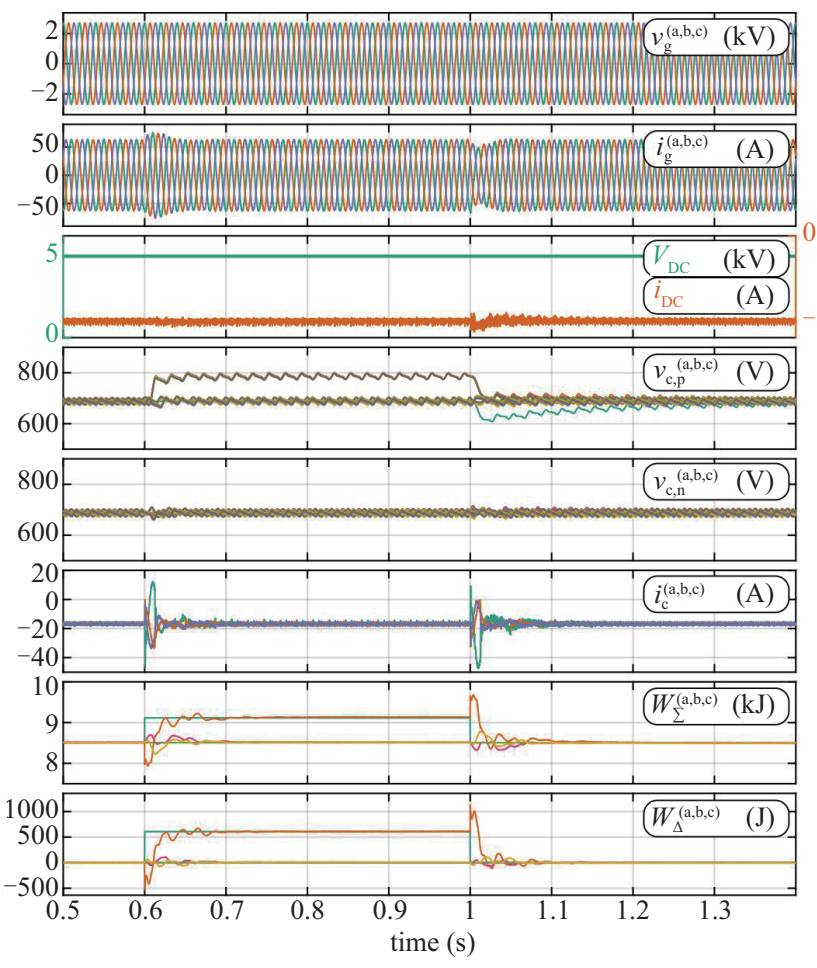

(a)

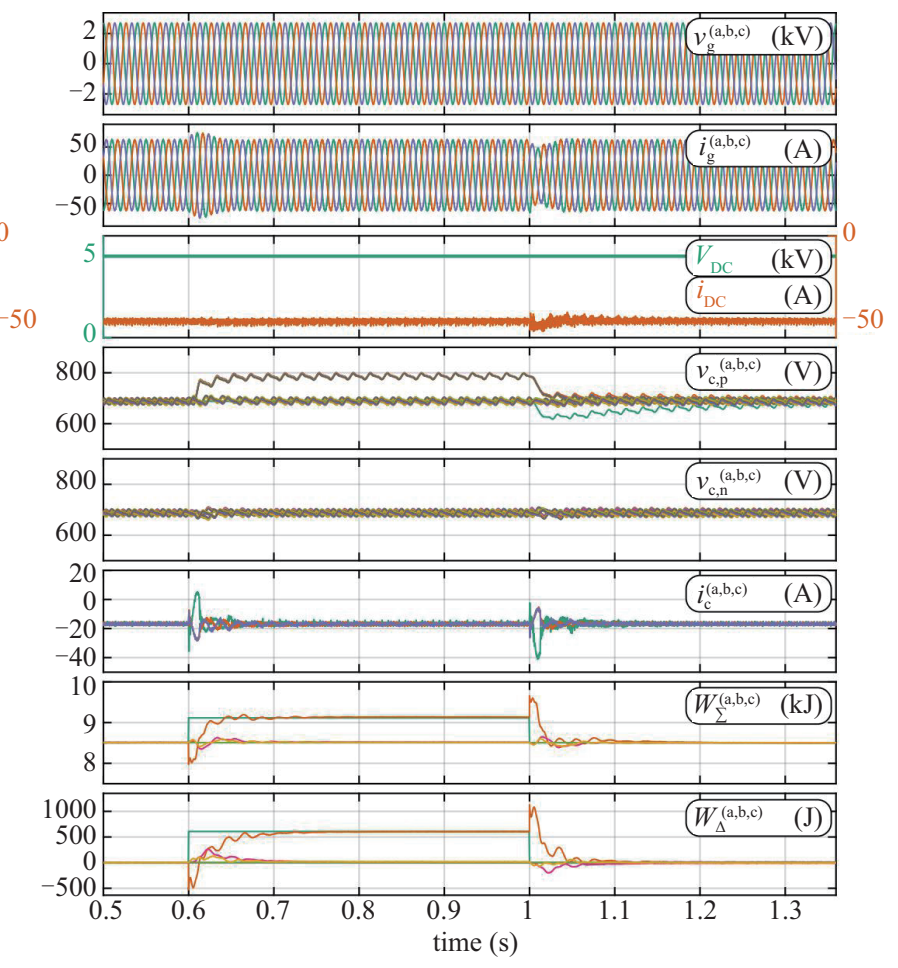

(b)

Fig. 13. MMC operation with one SM temporarily bypassed, emulating SM failure with (a) method I and (b) method II. Variables displayed from the top-bottom are as follows: $v_{\mathrm{g}}-3 \mathrm{PH}$ grid voltages; $i_{\mathrm{g}}-3 \mathrm{PH}$ grid (arm-differential) currents; $V_{\mathrm{DC}}-\mathrm{DC}$ terminals voltage; $i_{\mathrm{DC}}-\mathrm{DC}$ terminals current; $v_{\mathrm{c}, \mathrm{p} / \mathrm{n}}-$ individual SM voltages of the upper/lower arms; $i_{\mathrm{c}}-3 \mathrm{PH}$ arm-common currents; $W_{\Sigma}-3 \mathrm{PH}$ filtered arm-sum energies; $W_{\Delta}-3 \mathrm{PH}$ filtered arm-differential energies.

Modification of the original set of currents corresponds to the one resulting from the method II (33), with the only difference being that it is applied to DC variables in this case. In addition, owing to the per-phase approach, arm-sum energy control is valid for both balanced and unbalanced grid conditions. In both operating modes, corresponding terminal powers $\left(P_{\mathrm{DC}}\right.$ or $\left.P_{\mathrm{AC}}\right)$ are used as a feedforward signals, with the aim of improving the dynamic response of arm-sum energy controllers.

Moreover, as in case of arm-differential energy control with Method II, this current modification method is general, and can be applied to an MMC with an arbitrary number of phases [25], [26], or in case when the MMC arms are paralleled for the sake of power capability extension [27]. In the latter case each one of the sub-arms has a dedicated energy controller, and current modification principle described by (41) is applied to the current references of all sub-arms within one arm. The same holds for an MMMC, where each arm is equipped with a dedicated energy controller, with current modification method from (41) being applied to the groups of current references related to each one of the nodes.

In order to verify performance of arm-sum energy control, it is tested under the same scenario as arm-differential energy control. Fig. 12 illustrates the response of arm-sum energy control to the reference step changes of the two MMC arms. Arm-sum energy control presented proves to be effective, with the settling time of $50 \mathrm{~ms}$.

\section{Evaluation of the Direct Arm Energy Control}

It has been concluded that the arm-sum energy and armdifferential energy control actions are necessary parts of an MMC control structure. Moreover, due to internal nature of these control actions, generated currents should not be observed either at the AC, or at the DC terminals of an MMC. Figs. 6 and 8 showed the differences in current references being generated by the methods I and II, together with the performance of the arm-differential energy controllers as a response to the step change in arm energy references. In a similar way, Fig. 12 showed the performance of the arm-sum energy controller for the same step change in arm energy references.

As mentioned in the introduction, with failure of one or several SMs within an arm, remaining SMs are typically surcharged so that the voltage sum within an arm remains the same as in the pre-fault condition. Consequently, references for the arm-energies change, and so do the references of the arm-sum and arm-differential energies of the corresponding phase. To demonstrate the effectiveness of the proposed control concepts in case of failure of an SM, a single SM within the upper arm of phase A is temporarily bypassed at the time instant $t=0.6 \mathrm{~s}$ and put back into operation at $t=0.9 \mathrm{~s}$, thus emulating failed SM.

Simulation results for an MMC from Table I, with failed SM, are presented in Fig. 13 for both methods I and II. The results confirm theoretical considerations where method II is 


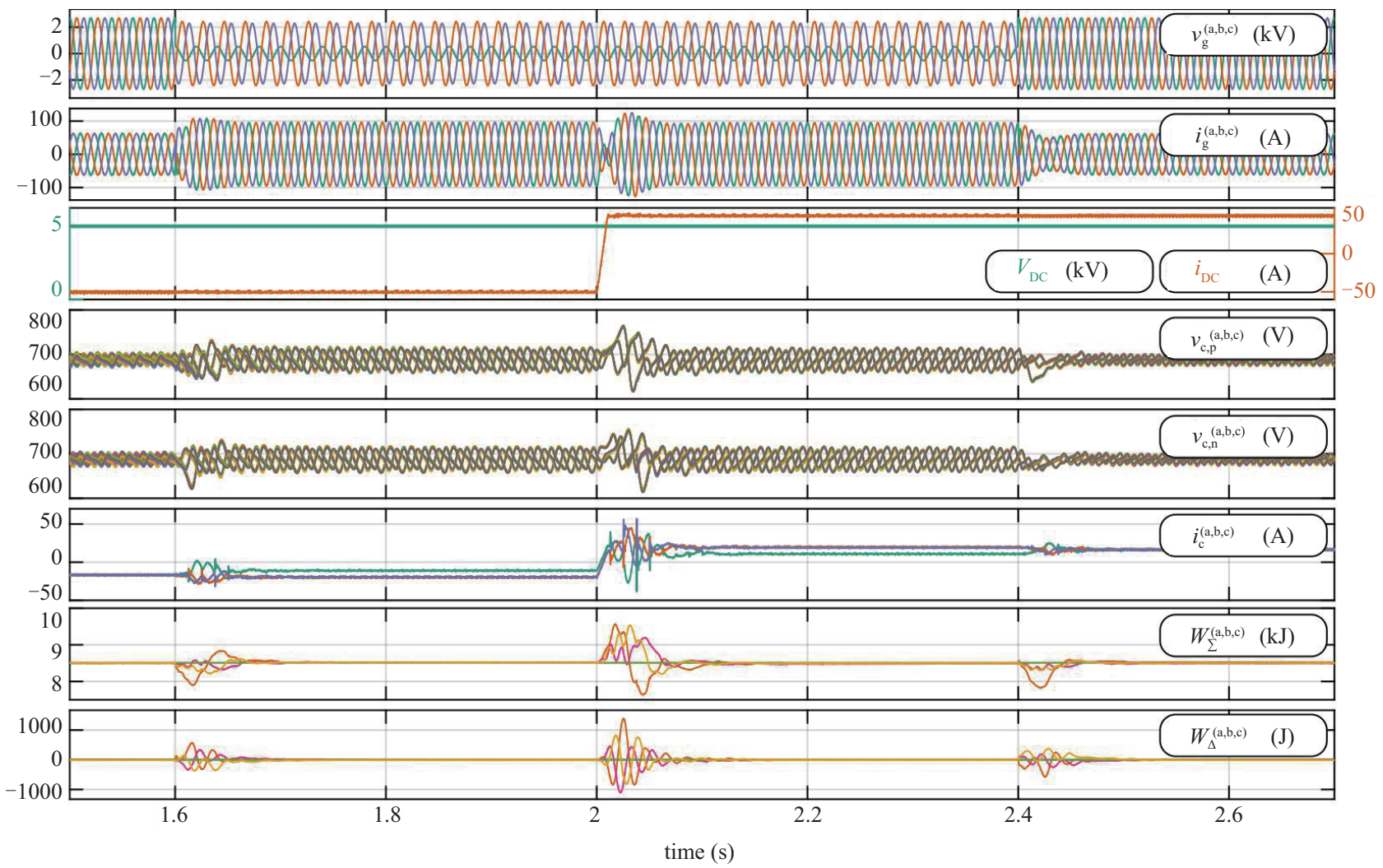

Fig. 14. Performance evaluation of the direct arm energy control under unbalanced grid conditions and power reversal in rectifier mode of operation. Singe phaseto-ground fault at $t=1.6 \mathrm{~s}$; active power reversal at $t=2 \mathrm{~s}$; fault clearance at $t=2.4 \mathrm{~s}$.

— Original space-vector trajectory

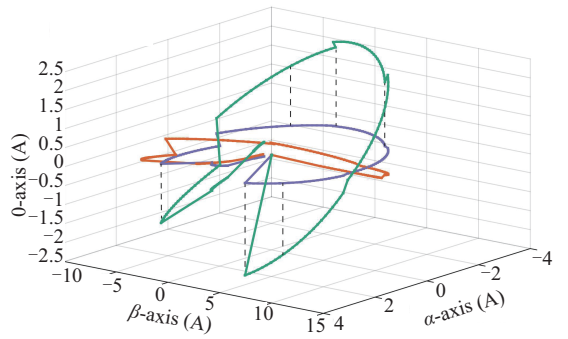

(a)
- Original space-vector $\alpha \beta$ projection

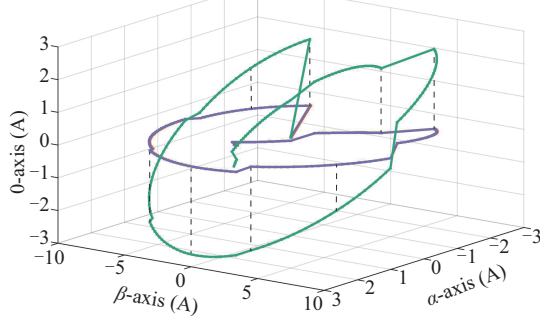

(b)
— Modified space-vector trajectory

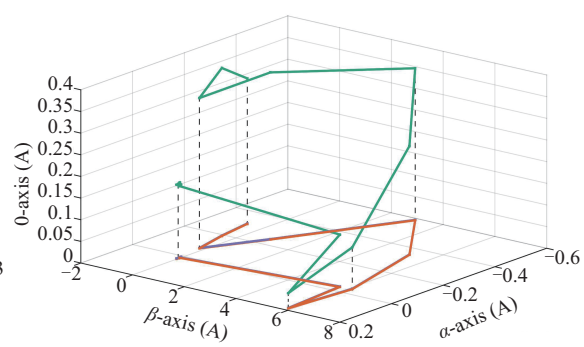

(c)

Fig. 15. Current space-vector before and after modification by: (a) arm-differential current modification method I, (b) arm-differential current modification method II and (c) arm-sum current modification.

shown to yield lower arm-common current for the energy control tasks. More importantly, it can be observed that both methods ensure successful tracking of the set energy references during the SM fault condition, and most importantly, enable proper operation of an MMC at its terminals.

Proposed control concepts are also applicable in case when MMC operates under unbalanced grid conditions. In order to verify its performance, $\mathrm{MMC}$ with the same parameters is tested under single-phase to ground short circuit occurring at $t=1.6 \mathrm{~s}$ and being cleared at $t=2.4 \mathrm{~s}$ (cf., Fig. 14). At time instant $t=2 \mathrm{~s}$, active power reference is reversed, however proposed energy control scheme maintains the arm-energies around defined values.
To illustrate the effects of the proposed methods on current reference modification, trajectories of the space-vectors of the reference current and modified current obtained from simulations, are illustrated in Fig. 15 in $\alpha \beta 0$ reference frame. What the three methods have in common is the fact that modified vectors are exempted from zero component. In case when method I is applied (cf., Fig. 15(a)), not only is the zero component removed, but also are the $\alpha \beta$ components modified. On the other side, method II (cf., Fig. 15(b)) preserves $\alpha \beta$ components of the original space-vector, thus representing the best possible approximation of the original space-vector under the given conditions. When it comes do arm-sum energy control (cf., Fig. 15(c)), proposed method of 
current modification shows the same behaviour as method II of arm-differential current modification, since they are based on the same principles. Results shown in (Fig. 15) verify the outcomes of the theory presented in this paper.

\section{CONCLUSION}

This paper presented control principles of the direct arm energy control. It has been shown that the energy content of each arm within an MMC can be controlled separately through the mechanisms called arm-sum energy control and arm-differential energy control in a 3PH MMC. Theoretical foundation behind arm-differential energy control has been thoroughly examined, two current vector modification methods have been introduced, together with novel approaches for their implementation. Method II has proved to be a method that poses the least current stress on the converter during the energy control actions, while still performs its control task. A straightforward way for its implementation to an MMC with an arbitrary number of phases has been proposed.

Arm-sum energy control has proven to be able to address the direct control over the arm-sum energies within an MMC, without influencing the converter terminal currents. Moreover, the method of current modification proposed in this paper, as part of arm-sum energy control, is generic, and thus can be applied to an MMC with an arbitrary number of phases, or to an MMC with paralleled arms, or within an MMMC, regardless of the nature of currents. In addition, simulation results illustrate that proposed control concepts show equally good performance under unbalanced grid conditions, as well as in case of a failed SM. That being said, the direct arm energy control can be perceived as a generalized approach to control energy content within the arms of various types of an MMC.

\section{ACKNOWLEDGEMENT}

This research project is part of the Swiss Competence Center for Energy Research SCCER FURIES of the Swiss Innovation Agency Innosuisse.

\section{REFERENCES}

[1] A. Lesnicar and R. Marquardt, "An innovative modular multilevel converter topology suitable for a wide power range," in Proceedings of 2003 IEEE Bologna Power Tech Conference Proceedings, Bologna, Italy, 2003 , pp. 6 , vol.3.

[2] H. Saad, S. Dennetière, J. Mahseredjian, P. Delarue, X. Guillaud, J. Peralta, and S. Nguefeu, "Modular multilevel converter models for electromagnetic transients," in IEEE Transactions on Power Delivery, vol. 29, no. 3, pp. 1481-1489, Jun. 2014.

[3] R. Marquardt, "Modular multilevel converters: State of the art and future progress," in IEEE Power Electronics Magazine, vol. 5, no. 4, pp. 24-31, Dec. 2018.

[4] M. Pereira, D. Retzmann, J. Lottes, M. Wiesinger, and G. Wong, "SVC plus: An MMC STATCOM for network and grid access applications," in Proceedings of 2011 IEEE Trondheim PowerTech, Trondheim, Jun. 2011, pp. $1-5$.

[5] Z. Guying, J. Daozhuo, and L. Xiaorang, "Modular multilevel converter for unified power flow controller application," in Proceedings of 2012 Third International Conference on Digital Manufacturing \& Automation, GuiLin, Jul. 2012, pp. 545-549.
[6] R. Xie and H. Li, "Fault performance comparison study of a dual active bridge (DBA) converter and an isolated modular multilevel DC/ DC (iM2DC) converter for power conversion module application in a breaker-less shipboard MVDC system," in IEEE Transactions on Industry Applications, vol. 54, no. 5, pp. 5444-5455, Sep.-Oct. 2018.

[7] J. Carr, D. Das, J. Li, J. Pan, S. Ebner, and O. Apeldoorn, "Modular multilevel converter for direct MVDC connection of offshore wind farms," in Proceedings of 2015 IEEE Energy Conversion Congress and Exposition (ECCE), Montreal, QC, Sep. 2015, pp. 976-982.

[8] A. Antonopoulos, L. Angquist, and H. Nee, "On dynamics and voltage control of the modular multilevel converter," in Proceedings of 2009 13th European Conference on Power Electronics and Applications, Barcelona, Sep. 2009, pp. 1-10.

[9] L. Harnefors, A. Antonopoulos, S. Norrga, L. Angquist, and H. Nee, "Dynamic analysis of modular multilevel converters," in IEEE Transactions on Industrial Electronics, vol. 60, no. 7, pp. 2526-2537, Jul. 2013.

[10] M. Guan and Z. Xu, "Modeling and control of a modular multilevel converter-based HVDC system under unbalanced grid conditions," in IEEE Transactions on Power Electronics, vol. 27, no. 12, pp. 4858-4867, Dec. 2012.

[11] S. Wang, G. P. Adam, A. M. Massoud, D. Holliday, and B. W. Williams, "Analysis and assessment of modular multilevel converter internal control schemes," in IEEE Journal of Emerging and Selected Topics in Power Electronics, vol. 8, no. 1, pp. 697-719, Mar. 2020.

[12] M. Hagiwara, R. Maeda, and H. Akagi, "Control and analysis of the modular multilevel cascade converter based on double-star chopper-cells (MMCC-DSCC)," in IEEE Transactions on Power Electronics, vol. 26, no. 6, pp. 1649-1658, Jun. 2011.

[13] K. Ilves, L. Harnefors, S. Norrga, and H. Nee, "Predictive sorting algorithm for modular multilevel converters minimizing the spread in the submodule capacitor voltages," in IEEE Transactions on Power Electronics, vol. 30, no. 1, pp. 440-449, Jan. 2015.

[14] S. Fan, K. Zhang, J. Xiong, and Y. Xue, "An improved control system for modular multilevel converters with new modulation strategy and voltage balancing control," in IEEE Transactions on Power Electronics, vol. 30, no. 1, pp. 358-371, Jan. 2015.

[15] P. Münch, D. Görges, M. Izák, and S. Liu, "Integrated current control, energy control and energy balancing of modular multilevel converters," in Proceedings of IECON 2010-36th Annual Conference on IEEE Industrial Electronics Society, Glendale, AZ, Nov. 2010, pp. 150-155.

[16] A. J. Korn, M. Winkelnkemper, P. Steimer, and J. W. Kolar, "Capacitor voltage balancing in modular multilevel converters," in Proceedings of 6th IET International Conference on Power Electronics, Machines and Drives (PEMD 2012), Bristol, Mar. 2012, pp. 1-5.

[17] J. Kolb, F. Kammerer, M. Gommeringer, and M. Braun, "Cascaded control system of the modular multilevel converter for feeding variable speed drives," in IEEE Transactions on Power Electronics, vol. 30, no. 1, pp. 349-357, Jan. 2015.

[18] M. Schroeder, F. Mahr, J. Jaeger, and S. H. Haensel, "Energy balancing in the modular multilevel converter under unbalanced grid conditions," in Proceedings of 2017 19th European Conference on Power Electronics and Applications (EPE'17 ECCE Europe), Warsaw, Sep. 2017, pp.1-10.

[19] J. Jung, S. Cui, Y. Lee, and S. Sul, "A cell capacitor energy balancing control of MMC-HVDC under the AC grid faults," in Proceedings of 2015 9th International Conference on Power Electronics and ECCE Asia (ICPE-ECCE Asia), Seoul, Jun. 2015, pp. 1-8.

[20] A. E. Leon and S. J. Amodeo, "Energy balancing improvement of modular multilevel converters under unbalanced grid conditions," in IEEE Transactions on Power Electronics, vol. 32, no. 8, pp. 6628-6637, Aug. 2017.

[21] M. Basić, S. Milovanović, and D. Dujić, "Comparison of two modular multilevel converter internal energy balancing methods," in Proceedings of 2019 20th International Symposium on Power Electronics(Ee), Novi Sad, Serbia, 2019, pp. 1-8, 2019.

[22] B. Li, Z. Xu, J. Ding, and D. Xu, "Fault-tolerant control of mediumvoltage modular multilevel converters with minimum performance degradation under submodule failures," in IEEE Access, vol. 6, pp. 11772-11781, 2018. 
[23] M. Winkelnkemper, A. Korn, and P. Steimer, "A modular direct converter for transformerless rail interties," in Proceedings of 2010 IEEE International Symposium on Industrial Electronics, Bari, Jul. 2010, pp. $562-567$.

[24] S. P. Engel and R. W. De Doncker, "Control of the modular multi-level converter for minimized cell capacitance," in Proceedings of the 2011 14th European Conference on Power Electronics and Applications, Birmingham, Aug. 2011, pp. 1-10.

[25] M. Daoud, A. Massoud, A. Elserougi, A. S. Abdel-Khalik, and S. Ahmed, "Sensor-less operation of hybrid boost modular multilevel converter for subsea multiphase medium voltage drives," in Proceedings of 2017 IEEE Southern Power Electronics Conference (SPEC), Puerto Varas, Dec. 2017, pp. 1-5.

[26] M. I. Daoud, A. A. Elserougi, A. M. Massoud, R. Bojoi, A. S. AbdelKhalik, and S. Ahmed, "Zero-/low-speed operation of multiphase drive systems with modular multilevel converters," in IEEE Access, vol. 7, pp. 14353-14365, 2019.

[27] S. Milovanovic and D. Dujic, "On facilitating the modular multilevel converter power scalability through branch paralleling," in Proceedings of 2019 IEEE Energy Conversion Congress and Exposition (ECCE), Baltimore, Oct. 2019, pp. 6875-6882.

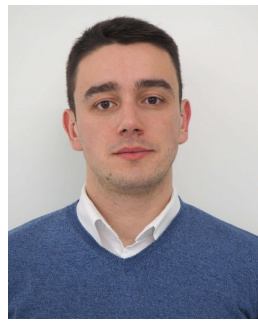

Milan Utvić received his B.Sc. and M.Sc. degrees from the School of Electrical Engineering, University of Belgrade, Serbia, in 2015 and 2017, respectively. From 2016 to 2017 he worked as a research assistant within the Department of Power Converters and Drive Systems at the University of Belgrade. In February 2018 he joined the Power Electronics Laboratory at École Polytechnique Fédérale de Lausanne, Lausanne, Switzerland, where he is currently pursing a Ph.D. degree. From April to June 2020, he was with ABB Traction Department in Turgi, Switzerland, for an industrial Ph.D. internship. His research interests include utility scale high power converters, power converters for renewables, electricdrives and traction power converters.

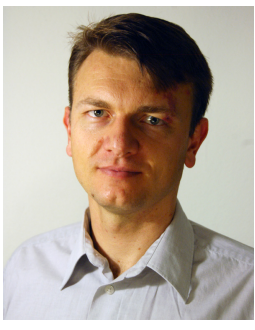

Dražen Dujić received the Dipl. -Ing. and M.Sc. degrees from the University of Novi Sad, NoviSad, Serbia, in 2002 and 2005, respectively, and the Ph.D. degree from the Liverpool John Moores University, Liverpool, U.K., in 2008, all in electrical engineering. From 2002 to 2006, he was with the Department of Electrical Engineering, University of Novi Sad as a Research assistant, and from 2006 to 2009 with Liverpool John Moores University as a Research Associate. From 2009 till 2013, he was with ABB Corporate Research Centre, Switzerland, as a Principal Scientist working on the power electronics projects spanning the range from low-voltage/power SMPS in below kilowatt range to medium voltage high-power converters in a megawatt range. From 2013 till 2014, he was with ABB Medium Voltage Drives, Turgi, Switzerland, as R\&D Platform Manager, responsible for ABB's largest IGCT based medium voltage drive ACS6000. He is currently with École Polytechnique Fédérale de Lausanne EPFL, Lausanne, Switzerland, as an Assistant Professor and the Director of the Power Electronics Laboratory. His current research interests include the areas of design and control of advanced high-power electronics systems and high performance drives. He has authored or coauthored more than 150 scientific publications and has filed 14 patents. He is an Associate Editor for IEEE Transactions on Industrial Electronics, IEEE Transaction on Power Electronics and IET Electric Power Applications. He has received the First Prize Paper Award by the Electric Machines Committee of the IEEE Industrial Electronics Society at IECON-2007. In 2018 he has received EPE Outstanding Service Award, and in 2014 the Isao Takahashi Power Electronics Award for outstanding achievement in power electronics. 